\title{
Perspectives
}

\section{Mechanisms of Viral Pathogenesis Distinct Forms of Reoviruses and Their Roles during Replication in Cells and Host}

\author{
Max L. Nibert, ${ }^{*}$ Deirdre B. Furlong, ${ }^{*}$ and Bernard N. Fields*:l \\ *Department of Microbiology and Molecular Genetics and ${ }^{\$}$ Shipley Institute of Medicine, Harvard Medical School, \\ and Departments of ${ }^{\ddagger}$ Pathology and "Medicine, Brigham and Women’s Hospital, Boston, Massachusetts 02115
}

Recent years have seen a resurgent interest in mechanisms by which viruses cause disease, or viral pathogenesis. Studies of viral pathogenesis often address the capacity of a virus to cause injuries to cells and tissues; yet, injuries are secondary consequences of many fundamental interactions that occur between virus and single cells, as well as between virus and host organism, during infection. For example the tropism of a virus, or its capacity to grow within particular cells in the host, reflects several of these interactions. Studies of more basic aspects of viral infection are therefore critical for understanding viral pathogenesis. We begin this perspective by introducing some general concepts in regard to the replication and structure of animal viruses.

Infections with mammalian reoviruses provide useful models for characterizing mechanisms of viral pathogenesis (reviewed in references 1 and 2). Mammalian reoviruses (reoviruses for short) are medium-sized nonenveloped viruses that have a genome consisting of 10 segments of double-stranded (ds) ${ }^{1}$ RNA. In humans, reoviruses are not linked definitively with any disease more severe than a mild enteric or respiratory illness, but in mice and rats they cause a number of disease syndromes involving major organs like brain (3) and heart (4). Studies with reoviruses demonstrate that facts obtained at multiple levels of inquiry can be integrated in the attempt to understand viral pathogenesis $(1,2)$. In the major part of this perspective, we describe how the structure of reoviruses relates to their strategy for replication in cells and host organisms and discuss several similarities in this regard between reoviruses and other animal viruses.

\section{Some general concepts regarding the replication and structure of animal viruses}

Defining the replication program of an animal virus. Animal viruses proceed through an orderly series of steps when replicating within cells (illustrated for reoviruses in Fig. 1). These steps are said to define the replication cycle of a virus. Because ani-

\footnotetext{
Address correspondence to Dr. Bernard N. Fields, Dept. of Microbiology and Molecular Genetics, Harvard Medical School, 260 Longwood Ave., Boston, MA 02115.

Received for publication 12 March 1991.
}

1. Abbreviations used in this paper: ds, double-stranded; ISVP, intermediate (or infections subviral particle; $M$ cell, microfold (or membranous) cell.

J. Clin. Invest.

(c) The American Society for Clinical Investigation, Inc. 0021-9738/91/09/0727/08 \$2.00

Volume 88, September 1991, 727-734 mal viruses infect complex host organisms in the natural world, however, considerations beyond the level of the cell are needed for identifying other steps in viral replication. In this perspective we wish to emphasize that viral growth in the host is a complex process, involving multiple cycles of replication within cells as well as other uniquely host-level steps (illustrated for reoviruses in Fig. 2). We therefore refer to the complete process as the replication program of an animal virus.

Features specific to viral replication in the host have been reviewed elsewhere $(5,6)$. Briefly, viruses infect their hosts in three general patterns: local, "local + systemic," and systemic infections (5). Local infection refers to growth of virus near its site of entry into the host, and systemic infection refers to growth at sites distant from the entry site. The pattern exhibited by reoviruses depends on numerous factors, but in infections by the enteric route, reoviruses often exhibit local + systemic infection $(7,8)$. Host-level steps in viral replication include entry into the host, primary replication, spread to distant sites, and secondary replication (6) (Fig. 2). Viral tropism is reflected in both primary and secondary replication but is often discussed in regard to secondary replication because at that step virus grows in only some of the many cells it encounters in the host (Fig. 2).

Two additional steps that can be identified as essential to viral replication in the host are release of virus from the infected host and survival in the environment between hosts (9) (Fig. 2). These steps highlight the fact that a virus must be capable of spreading between hosts to survive in the natural world. Survival and spread between hosts are formally similar to survival and spread between sites of replication within the host: outside the host, virus must contend with the barrier of distance and physicochemical challenges like drying and radiation; inside the host, there are anatomical barriers and challenges like those imposed by the host immune response. The relevance of studying viral survival in the face of specific challenges is not immediately apparent when thinking only about viral replication in isolated cells.

A schematic description of the structure of animal viruses. The design of a viral particle is simple, but it permits the virus to participate in a series of complex interactions with cells and hosts. Although it is easy to recognize that viral structure has been molded by requirements for growth in cells, it is likely that distinct requirements for growth in the host have also contributed selective pressures determining viral structures. The latter pressures include, but are not limited to, those posed by the host response.

We prefer to consider that the structural components with which mature viral particles are built fall into two functional categories: the delivery system and the payload (Fig. 3). Components of the delivery system initiate the replication cycle by 


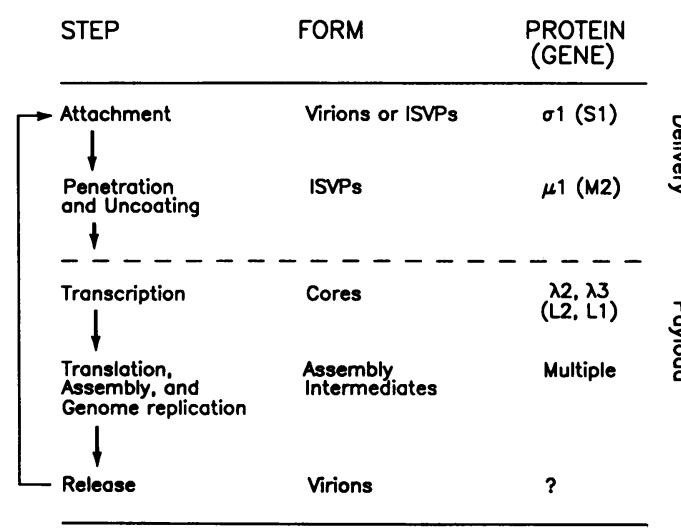

Figure 1. Replication of reoviruses in cells (replication cycle). Indicated are identifiable steps in the replication of reoviruses in cells, the particular viral forms (virions, ISVPs, and cores) that are associated with these steps, and individual viral proteins that have specific functions during these steps. The $\sigma 1$ protein is the cell-attachment protein that binds viral particles to specific receptors on the cell surface. The $\mu 1$ protein is thought to mediate the penetration of cell membranes by viral particles, and its removal from particles permits activation of the viral transcriptase. The $\lambda 2$ and $\lambda 3$ proteins are known components of the viral transcriptase apparatus: $\lambda 2$ is the guanylyltransferase and $\lambda 3$ is a catalytic component of the RNA-dependent RNA polymerase. Attachment and penetration are effected by the delivery system contained in both virions and ISVPs as indicated at right. Cores are proposed to represent the payload of reoviruses, whose immediate function after penetration consists of transcribing the viral mRNAs.

delivering the payload to an intracellular site (cytoplasm or nucleus) within cells appropriate for replication; components of the payload then participate in the intracellular steps of replication. As might be predicted from the order of their functions, the delivery system is located more externally in viral particles and the payload more internally. Thus, the basic organization of a viral particle has a simple logic (Fig. 3).

The two best known delivery functions are attachment of virus to receptors on the cell surface and penetration of viral components into the cytoplasm (Fig. 1). Cytoplasmic penetration is usually characterized by loss of external components (uncoating) and concomitant introduction of internal components into the cytoplasm. Viruses that replicate within the nucleus of cells can be considered to have additional delivery functions that permit viral components to be introduced into the nucleus. The fact that animal viruses replicate within complex hosts increases the complexity of functions required of the delivery system. In particular, viral delivery during replication in the host includes important steps that precede attachment: because delivery-system components occupy external positions in viral particles, they are the primary determinants of viral survival in the face of both environmental and host challenges.

The payload carried inside a viral particle consists of information-bearing elements (genome) plus associated components which protect the genome from degradation and supply functions for initiating intracellular steps of replication (Fig. 1). In the latter capacity these components may aid in translating or transcribing the genome, integrating it into the host DNA, or keeping it quiescent. They may also interact with cellular factors and thereby enhance or regulate viral replication. Al-

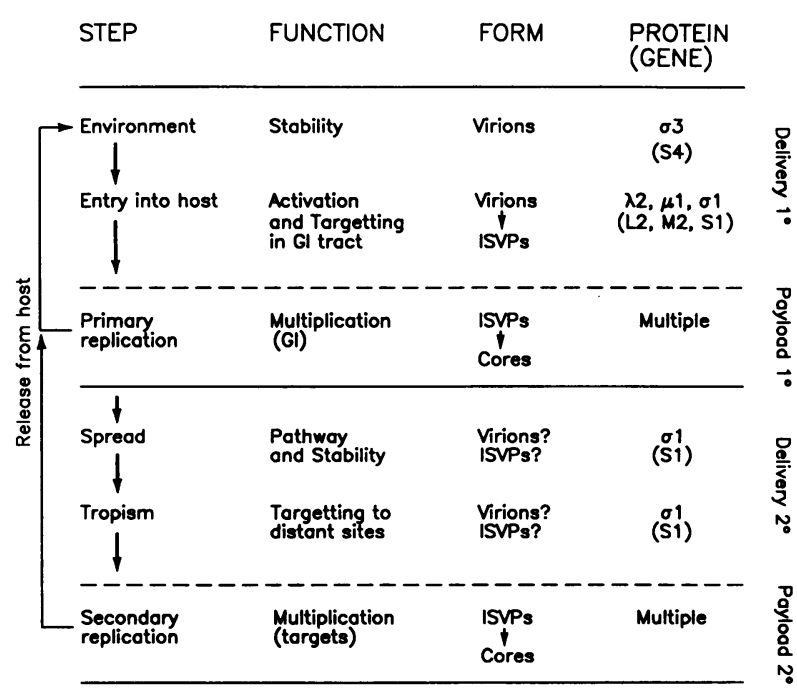

Figure 2. Replication of reoviruses in the host (replication program). This figure parallels Fig. 1. Indicated are steps in the replication of reoviruses in the host after infection by the enteric route and a description of viral functions associated with these steps. Also indicated are the particular viral forms associated with the steps and individual viral proteins that have been indicated by genetic and biochemical studies to play specific roles during replication in the host (described in the text). Replication in the host is schematized as consisting of two rounds of replication: primary replication associated with the portal of entry into the host (gastrointestinal tract in this case) and secondary replication at distant sites. Both rounds of replication have functions of the viral delivery system and viral payload associated with them as indicated at right. An important aspect of replication in the host are delivery functions that precede attachment to the cells in which viral multiplication occurs.

though payload components act intracellularly, considerations of replication in the host are important for understanding their functions. For example, nonspecific host-response proteins (e.g., interferon) can stimulate cellular activities that affect intracellular steps in viral replication.

\section{Relating structure to replication in reoviruses}

Structure of reovirus virions. Our current view of the structure of reoviruses has been obtained from biochemical studies and from conventional studies with the electron microscope (also reviewed in reference 10). The 10 dsRNA gene segments of reoviruses encode 11 proteins, eight of which are included in

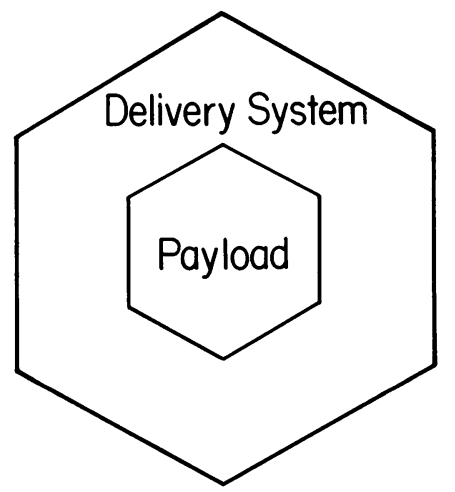

Figure 3. A schematic description of the structure of animal viruses. The more external components of viral particles constitute a delivery system for localizing virus to appropriate sites within appropriate cells for viral replication. The more internal components of viral particles constitute the payload that is delivered to the inside of cells and initiates the intracellular steps of viral replication. 
mature virions. Copies of the eight structural proteins are arranged in virions such that they surround the dsRNA genome in two concentric layers, or capsids, each appearing to have icosahedral symmetry (11-13). The internal capsid includes two proteins present in $>100$ copies per virion $(\lambda 1$ and $\sigma 2$, encoded by the L 3 and S 2 genes, respectively). Two minor proteins present in $\leq 12$ copies per virion ( $\lambda 3$ and $\mu 2$, encoded by L1 and M1) may also be associated with the internal capsid. The external capsid includes two major proteins present in $\geq 600$ copies per virion ( $\mu 1$ and $\sigma 3$, encoded by M2 and S4) and a minor protein present in $\leq 60$ copies per virion $(\sigma 1$, encoded by S1). The $\mu 1$ protein has been shown to undergo proteolytic cleavage during assembly so that it is mostly represented in virions by its amino- and carboxyl-terminal fragments, $\mu 1 \mathrm{~N}$ and $\mu 1 \mathrm{C}(14,15)$. Another protein present in 60 copies per virions ( $\lambda 2$, encoded by $L 2)$ is thought to span both capsid layers.

The arrangement of components in the internal capsid of reoviruses is poorly understood. It is likely that the $\lambda 1$ and $\sigma 2$ proteins, and perhaps a portion of the $\lambda 2$ protein, form the basic shell of the internal capsid (13). Recent evidence indicates that $\lambda 1$ is a zinc metalloprotein $(16,17)$. Pentamers of the $\lambda 2$ protein are located at the icosahedral vertices $(11,13,18)$, and the minor proteins $\lambda 3$ and $\mu 2$ may also be located there (4; Nibert, M., and E. Brown, unpublished data).

More is known about the external capsid of reoviruses (cover picture). Components of the external capsid appear to be arranged in a $T=13 \mathrm{l}$ icosahedral lattice $(11,12)$. The basic shell of the external capsid is formed by the $\mu 1$ protein, except at the icosahedral vertices where it is substituted by the pentamers of $\lambda 2(11,13,18)$. The amino terminus of $\mu 1$ and that of its amino-terminal cleavage fragment $\mu 1 \mathrm{~N}$ are modified by an amide-linked myristoyl $\left(C_{14}\right.$ saturated fatty acyl) group so that there are a large number $(\geq 600)$ of these fatty acyl chains in the external capsid (15). The $\sigma 3$ protein decorates the outer surface of the external capsid $(12,19)$ and is present in virions in the same number of copies as $\mu 1$ (or $\mu 1 \mathrm{~N}+\mu 1 \mathrm{C}$ ) (14). Because $\sigma 3$ is a metalloprotein that binds approximately one zinc ion per molecule, there are also a large number $(\geq 600)$ of zinc ions in the external capsid (16). The $\sigma 1$ protein is located at the icosahedral vertices $(19,20)$ and occurs as an oligomeric (probably tetrameric) complex of $\sigma 1$ subunits $(21,22)$. It may be capable of assuming both a folded and an extended conformation in viral particles (19). In its extended form $\sigma 1$ projects out as much as $500 \AA$ from the surface of particles, to which it remains attached by a region of sequence near its amino terminus $(19,23)$. The head-and-tail morphology of the extended $\sigma 1$ complex $(19,22,24)$ has been correlated with its amino acid sequence, indicating that the tail domain is formed from alternating regions of $\alpha$-helical coiled-coil and cross- $\beta$ sandwich motifs of supersecondary structure $(22,25)$.

The three-dimensional structures of several small nonenveloped animal viruses have been defined at the atomic level using $x$-ray crystallographic techniques (e.g., reference 26). We have begun to apply these techniques to reoviruses (27); however, completion of such studies may require significant time because reoviruses are larger and have a more complex structure than previously studied viruses. Other techniques, namely electron cryomicroscopy and three-dimensional image reconstruction, have been used to define the structures of several viruses at lower resolution (28). Studies applying the latter techniques to reoviruses should soon add much to our understand- ing of their structure (Dryden, K., D. Furlong, K. Coombs, B. Fields, and T. Baker, unpublished data).

Subviral (subvirion) particles of reoviruses: ISVPS and cores. The reoviruses have an interesting property which is likely a reflection of their multilayered structure: in addition to virions, there are two types of stable "subviral particles" (cover picture). The subviral (better called subvirion) particles of reovirus have been given the names "ISVPs" (intermediate, or infectious, subviral particles) and "cores," and each can be derived from virions by treatment with purified proteases in vitro (29-31). Which of the two types of subvirion particle results as the end-product of protease treatment is determined by the treatment conditions. Subvirion particles are also generated during replication of reoviruses in cells and host organisms as will be discussed in detail below.

Both ISVPs and cores are formed by removing more external proteins of virions (cover picture). ISVPs differ from virions in that they lack one of the outermost proteins, $\sigma 3$. The $\sigma 3$ protein in virions is very susceptible to proteolytic cleavage and is degraded rapidly during protease treatment $(29,32,33)$. In addition, during a protease treatment that generates ISVPs, the $\mu 1$ protein and $\mu 1 \mathrm{C}$ protein fragment are specifically cleaved to generate large amino-terminal subfragments (named $\mu 1 \delta$ and $\delta$, respectively) $(14,15,29,30,31,34)$ and a small carboxyl-terminal subfragment (Nibert, M., unpublished data), which remain attached to ISVPs. Lastly, the $\sigma 1$ protein, which also remains attached to ISVPs, may undergo a transition from folded to extended conformation during the generation of ISVPs with many reovirus strains (19; Furlong, D., unpublished data). Cores differ from virions and ISVPs in that they lack all external-layer proteins but $\lambda 2(29-31)$. The $\lambda 2$ protein remains attached to cores and forms the wide "spike" seen at each of the 12 core vertices (13).

A simplified summary of the properties of ISVPs and cores is as follows. ISVPs, like virions, are infectious when absorbed to cells but are incapable of transcribing full-length mRNA in vitro; cores have negligible infectivity when absorbed to cells but are capable of transcribing full-length mRNA from all 10 viral gene segments in vitro $(29,31)$. Thus, the ISVP-to-core conversion is characterized by loss of infectivity and acquisition of transcriptase activity. The transcriptase is probably latent in virions and ISVPs because of steric constraints imposed by external-layer proteins such as $\mu 1 \mathrm{C}(29,32,35,36)$. Some disagreement in the literature as to properties of ISVPs (30) might be explained by observations that $(a)$ the $\sigma 1$ protein of some reovirus strains is cleaved during the generation of ISVPs, with an accompanying decrease in particle infectivity (37; Nibert, M., T. Dermody, and D. Bodkin, unpublished data), and (b) ISVPs are less stable than virions under several sets of conditions and may undergo a spontaneous change such that they become transcriptionally active if not handled with appropriate care (32).

Role of ISVPs and cores in the infection of cells by reoviruses. When virions are used to infect cells in culture, the $\sigma 3$ and $\mu 1 \mathrm{C}$ proteins of infecting virions are cleaved in similar ways as during generation of ISVPs in vitro $(33,38-40)$. These cleavages may be initiated by proteases inside endosomes or lysosomes, which virions enter after attachment and endocytosis (33). Work from our laboratory has not only confirmed that these cleavages occur but also suggested that they are essential for reovirus replication in cells (Fig. 1). When virions are used to infect cells, ammonium chloride blocks growth of virus by 
inhibiting an early step in replication $(33,41,42)$; furthermore, it inhibits proteolytic processing of virion proteins that occurs early in infection but fails to block growth of virus when ISVPs generated in vitro are used to infect cells $(33,42)$. These findings have led us to suggest that processing of viral proteins by acid-dependent cellular (endosomal or lysosomal) proteases is the step in reovirus replication that is sensitive to ammonium chloride (33). Our current understanding is that only proteolytically processed particles can perform the next step in reovirus entry, i.e., penetration of a cellular membrane; thus, proteolysis appears to be an essential step during reovirus replication in cells. Similar suggestions have been made by other investigators $(40,43)$. We are not yet certain which of the unique structural features of ISVPs (degraded $\sigma 3$, cleaved $\mu 1$ and $\mu 1 \mathrm{C}$, and extended $\sigma 1$; cover picture) are essential for their capacity to resist inhibition by ammonium chloride. In addition, another explanation for the available data might be that a pH-dependent conformational change in the external protein layer of virions is required during infection of cells but is not required if the external layer has been altered by prior treatment with protease.

Whether cores are generated at a succeeding step in reovirus replication in cells is somewhat uncertain. Because cores but not ISVPs are capable of transcribing full-length mRNA, it seems likely that a core is the transcriptionally active form of virus that gains access to the cytoplasm during penetration (15) (Fig. 1). Consistent with this suggestion is the finding that cores are infectious when introduced into the cytoplasm by microinjection (44). But how do ISVPs in endosomes or lysosomes become cores in the cytoplasm? Models suggest that external components of ISVPs undergo changes in conformation such that they become capable of interacting directly with a cellular membrane and thereby introduce themselves $(33,40,43)$ or uncoated cores (15) into the cytoplasm. Another model suggests that cores generated from infecting particles do not completely enter the cytoplasm but assume positions still inside endosomes or lysosomes such that they can transcribe viral mRNA through the $\lambda 2$ spikes and into the cytoplasm (33). The higher concentration of potassium inside versus outside cells may contribute to the uncoating of reoviruses $(32,40)$. Obtaining a better description of reovirus penetration and uncoating is the goal of current work. For example, recent findings suggest that the myristoylated cleavage fragments of $\mu 1$ are components of ISVPs that may be capable of interacting directly with membranes $(15,45)$.

The delivery-and-payload description of viral structure provides an interesting way to summarize our views about the subvirion particles of reoviruses. Cores are likely to represent the reovirus payload (Figs. 1 and 3, cover picture). Even though most core proteins are constrained in an icosahedral capsid structure, they perform usual functions for a viral payload: they effect all enzymatic activities needed to produce capped mRNA from each dsRNA gene segment and protect the genome from degradation in the cytoplasm. Because dsRNA is a potent inducer of interferon and an essential cofactor for some of the interferon-stimulated antiviral activities in cells, core proteins may also help to keep the reovirus genome from generating a high-level interferon-related antiviral response. Specific roles have been assigned to the $\lambda 2$ and $\lambda 3$ proteins in production of capped viral mRNA by cores: $\lambda 2$ is the guanylyltransferase that aids in synthesizing a $5^{\prime}$ cap on each viral mRNA $(46,47)$ and $\lambda 3$ is a catalytic component of the RNA- dependent RNA polymerase $(35,48)$. Specific roles have yet to be assigned to the $\lambda 1, \mu 2$, and $\sigma 2$ proteins; however, $\lambda 1$ and $\sigma 2$ can each bind dsRNA (17), and $\lambda 1$ contains a nucleotide-binding sequence motif suggesting that it also performs an enzymatic function (18). The core is delivered to the appropriate intracellular site for replication by the viral delivery system, which is contained in both virions and ISVPs (Figs. 1 and 3, cover picture). The delivery system consists of the $\sigma 1$ protein, which is responsible for attachment to specific carbohydrate and/or protein receptors on the surface of cells (49-51), and other external-layer proteins (certainly $\mu 1$ or its cleavage fragments, but perhaps $\lambda 2$ and $\sigma 1$ as well), which act during penetration. We favor a description of early events in infection of cells by reoviruses in which the ISVP-to-core conversion is analogous to uncoating that accompanies penetration of enveloped viruses into the cytoplasm.

Role of ISVPs and virions in infection of the host by reoviruses. We have so far suggested roles for both ISVPs and cores during reovirus replication in cells. Might subvirion particles also play specific roles during replication in the host? Recent work from our laboratory has addressed this question (Fig. 2). When virions are perorally inoculated into neonatal mice, they are converted to ISVPs in the lumen of the small intestine, presumably by the action of pancreatic proteases (52). The generation of ISVPs in the gut lumen is blocked after peroral treatment with serine protease inhibitors; furthermore, mice treated with protease inhibitors before inoculation with virions show little or no growth of virus in small intestine, whereas mice treated identically but inoculated with ISVPs generated in vitro show normal growth of virus (53). These findings suggest that generation of ISVPs in the lumen of the gut (i.e., an extracellular location) is an important (perhaps necessary) step for infection by that route (Fig. 2).

We have yet to identify the step at which ISVPs can function more effectively than virions in the gut, but they may be important for attachment to or transport across $\mathbf{M}$ (microfold) cells (via which reoviruses can cross the intestinal epithelium and gain access to the inside of the host [54]) or for attachment to or penetration of the first cells in which virus grows (perhaps intestinal epithelial cells [55] or macrophages in the Peyer's patches [8]). In addition, we do not yet know whether the virion-to-ISVP conversion occurs in other extracellular locations at later steps in replication in the host (e.g., during spread in nerves or in the bloodstream [56]) or after infection by different routes (e.g., respiratory) (Fig. 2). Studies in cultured cells indicate that the virion-to-ISVP conversion of reoviruses may occur within endosomes or lysosomes of some cells that they infect so that proteolytic cleavage in an extracellular location may not be essential for infection of all cells or tissues in the host by reoviruses.

The observation that the virion-to-ISVP conversion occurs in the lumen of the murine small intestine has led us to consider that virions (versus ISVPs or cores) have a specific role in the natural sequence of enteric infections by reoviruses: virions are the stable form of virus that is best suited to pass between hosts (Fig. 2, Table I). A role for virions in this case should precede the conversion step generating ISVPs, i.e., to occur in the large intestine before release from the original host, in the external environment, or in the upper gastrointestinal tract (including stomach) of the new host. These are sites of such relative harshness that virus is likely to require its maximal stability for surviving there (9). The $\sigma 3$ protein, which virions contain 
Table I. Proposal for How Different Forms of Viruses Relate to Their Replication Programs

\begin{tabular}{|c|c|c|c|}
\hline $\begin{array}{l}\text { Group } \\
\text { of viruses }\end{array}$ & $\begin{array}{l}\text { "Extrahost" } \\
\text { (stable) } \\
\text { form }\end{array}$ & $\begin{array}{l}\text { "Intrahost" } \\
\text { (activated) } \\
\text { form }\end{array}$ & $\begin{array}{l}\text { Intracellular } \\
\text { (uncoated) } \\
\text { form }\end{array}$ \\
\hline Reoviruses & Virions & ISVPs & Cores \\
\hline Rotaviruses & $\begin{array}{l}\text { Double-shelled } \\
\text { particles with } \\
\text { uncleaved VP4 }\end{array}$ & $\begin{array}{l}\text { Double-shelled } \\
\text { particles with } \\
\text { cleaved VP4 }\end{array}$ & $\begin{array}{l}\text { Single-shelled } \\
\text { particles }\end{array}$ \\
\hline Coronaviruses & $\begin{array}{l}\text { Enveloped } \\
\text { particles with } \\
\text { uncleaved E2 }\end{array}$ & $\begin{array}{l}\text { Enveloped } \\
\text { particles with } \\
\text { cleaved E2 }\end{array}$ & $\begin{array}{l}\text { Nucleocapsid } \\
\text { components }\end{array}$ \\
\hline $\begin{array}{l}\text { Orthomyxo- } \\
\text { viruses }\end{array}$ & $\begin{array}{l}\text { Enveloped } \\
\text { particles with } \\
\text { uncleaved HA }\end{array}$ & $\begin{array}{l}\text { Enveloped } \\
\text { particles with } \\
\text { cleaved HA }\end{array}$ & $\begin{array}{l}\text { Nucleocapsid } \\
\text { components }\end{array}$ \\
\hline
\end{tabular}

but ISVPs do not, has been implicated in the capacity of reoviruses to survive a number of chemical and physical challenges $(32,57)$; thus, the $\sigma 3$ protein may have a function in increasing the stability of virions. This stabilizing function of $\sigma 3$ is consistent with the delivery-and-payload description of viral structure, which assigns an important role in maintaining viral stability to components of the delivery system. In fact, studies have identified two other delivery-system components, $\mu 1$ and $\sigma 1$, as determinants of the survival of reoviruses in the face of different challenges (57). Because virions also differ from ISVPs in having $\mu 1$ and $\mu 1 \mathrm{C}$ proteins that have not been cleaved near their carboxyl termini and a $\sigma 1$ protein that is perhaps folded against the particle surface, these structural differences may also contribute to a difference in stability between virions and ISVPs. It seems worth commenting in this perspective that the specialized role which we are now suggesting for virions of reoviruses became evident only upon considering steps that are required for viral replication in the host organism; the concept of viral stability in extracellular and "extrahost" locations has little relevance to studies of viral replication in cultured cells.

The virions of reoviruses may be functionally analogous to specialized forms of other organisms. For example virions are similar to the spores formed by some bacteria to enhance their survival under harsh conditions. Changes in bacterial form during entry into the host may be a common phenomenon (58). The protective function of $\sigma 3$ protein in virions of reoviruses also suggests an analogy between $\sigma 3$ and polyhedron proteins of some insect viruses (e.g., cytoplasmic polyhedrosis viruses). The polyhedron proteins protect viral particles in the environment but are removed consequent to the alkalinity of the insect gut before infection of cells (59); this description is remarkably similar to ours of $\sigma 3$. We will expand this discussion below to suggest how other animal viruses might possess distinct forms analogous to the virions and ISVPs of reoviruses.

\section{Specific comparisons between reoviruses}

\section{and other animal viruses}

Structural similarities in reoviruses and some other nonenveloped viruses. Lessons learned in regard to the structure of reoviruses may not apply to reoviruses alone. Two other groups of nonenveloped animal viruses having dsRNA genomes, rotaviruses and orbiviruses, are included with reoviruses in the family Reoviridae. Although these different groups of dsRNA vi- ruses are not closely related at the level of their primary sequences, they share several similarities of structure. Rotaviruses and orbiviruses, like reoviruses, have external protein layers arranged in a $T=13 l$ icosahedral lattice $(12,28)$. Recently rotaviruses were shown to have a protein (VP4) that extends from the viral surface, albeit for a shorter distance away from the surface and in different symmetry-related positions than the $\sigma 1$ protein in reoviruses (28). In addition, stable subvirion particles can be isolated from virions of both rotaviruses and orbiviruses, which suggests not only that these viruses possess distinct inner capsid layers but also that subvirion particles may play specific roles in their replication programs as with reoviruses.

Reoviruses also share structural similarities with more divergent groups of nonenveloped animal viruses. Adenoviruses are dsDNA viruses that are similar to reoviruses in size and in having an external capsid formed primarily by one protein (hexon) but substituted at the icosahedral vertices by another protein (penton). In addition, they have a distinct protein (fiber) that projects from the surface of virions at the vertices. Although adenovirus virions contain other internally located proteins, it is not known if any are organized into an icosahedral inner capsid as in reoviruses. The $\mu 1$ protein of reoviruses shares similarities with capsid proteins of another group of nonenveloped viruses, the picornaviruses. Like the $\mu 1$ protein, the capsid polyproteins of picornaviruses are myristoylated at their amino termini (60) and undergo a proteolytic cleavage near their amino termini to generate a small myristoylated amino-terminal fragment (named $\mu 1 \mathrm{~N}$ and VP4 in reoviruses and picornaviruses, respectively) (26). This cleavage is an autocatalytic one in picornaviruses (i.e., results from structural features inherent to the picornavirus particle), and limited sequence similarity across the cleavage sites suggests that aminoterminal cleavage of $\mu 1$ may also be autocatalytic (15).

Structural parallels in reoviruses and other viruses might indicate that similar structural designs have been selected along more than one evolutionary line for building nonenveloped viruses of similar complexity or functional requirements. For example, the $T=13$ arrangement of external-layer components may represent a good solution to the problem of building virions with concentric capsid layers. In addition, the extended proteins-reovirus $\sigma 1$, adenovirus fiber, and rotavirus VP4are all hemagglutinin (HA) proteins, and reovirus $\sigma 1$ and adenovirus fiber are the cell-attachment proteins of their viruses. In regard to myristoylation, myristoyl groups are important for the assembly and final structure of picornaviruses as well as for their capacity to penetrate cell membranes $(60)$, and the same is likely true for reoviruses.

Activational cleavages of surface proteins of other viruses. Although the virion-to-ISVP conversion of reoviruses is referred to as uncoating in the literature (e.g., reference 33), this terminology is imprecise. For other viruses, "uncoating" refers to loss of components from viral particles accompanying penetration. Specifically, components of the delivery system are lost during uncoating, and uncoated viral components are no longer infectious when applied to cells because they lack capacities for binding and penetration. The virion-to-ISVP conversion of reoviruses, however, occurs at a time preceding penetration. In addition, ISVPs are infectious because they still contain the essential delivery-system components $\sigma 1$ and $\mu 1$ (or $\mu 1 \mathrm{~N}+\mu 1 \mathrm{C}$ ). Thus, the virion-to-ISVP conversion of reoviruses does not represent uncoating as defined for other viruses. 
The virion-to-ISVP conversion of reoviruses appears more similar to the activational cleavage of surface proteins that is common to numerous viruses. For example, fusion proteins of many enveloped viruses, like the HA protein of orthomyxoviruses, undergo activational cleavages which are not required for assembly of viral particles, are effected by nonvirally encoded proteases, are important (or essential) for the capacity of those viruses to penetrate into the cytoplasm, and occur at a time preceding penetration so that activated particles remain infectious. In all these respects the activational cleavage of fusion proteins is similar to the virion-to-ISVP conversion of reoviruses.

Given that viruses naturally replicate within host organisms, it is important to define when activation occurs during viral replication in the host. In this perspective we have suggested that reoviruses exhibit an elegant but previously unrecognized strategy, in which activation can occur specifically at the time of entry into the host and changes an environmentally stable form of virus (virions) to a distinct, less stable but more infective form (ISVPs). In the case of other viruses, activational cleavage can be a determinant of virulence during infection of the host, but the timing of activation during replication in the host is poorly defined. Findings with reoviruses prompt us to propose that other viruses share the strategy of having a viral form (with uncleaved surface proteins) that is designed for survival in the environment; thus, one particular step in other viral replication programs at which activational cleavage of viral surface proteins is predicted to occur is at the time of entry into the host (Table I).

This strategy is easiest to envisage for enteric viruses because of extracellular proteases in the enteric tract that might activate the uncleaved, stable forms of viruses. For example, some coronaviruses can infect hosts via the enteric route; thus, coronavirus particles with uncleaved E2 protein might represent the stable form that passes between hosts and is activated for infection by cleavage of E2 protein in the gut of the new host (Table I). Rotaviruses are examples of nonenveloped enteric viruses having a surface protein (VP4) whose cleavage enhances penetration; thus, particles with uncleaved VP4 might represent the stable form of rotaviruses, which is activated by intestinal cleavage of that protein (Table I). Cleavage of surface proteins of many respiratory viruses, such as the HA protein of orthomyxoviruses and $F$ protein of paramyxoviruses, may reflect a similar strategy (Table I); however, in the case of respiratory viruses, the source of proteases that might effect the activation of uncleaved viral particles is an important question. Because orthomyxoviruses commonly infect avian species by the enteric route, the strategy is more readily envisaged for orthomyxoviruses during infection of birds.

There appears to be a need for balance between the "instability" of the viral delivery system necessary for viral particles to uncoat during penetration and its stability necessary for them to survive in environments between hosts. This need for balance in properties of the viral delivery system represents a fundamental problem that must be solved by all viruses. Proteolytic cleavage of delivery-system components represents a possible solution to this problem by allowing viral particles to be changed between more and less stable forms. In the case of orthomyxoviruses, the sensitivity of HA protein to activational cleavage affects virulence in the host: the more readily cleaved is the HA protein, the more virulent is the strain (61). Yet if viruses with cleaved HA protein have decreased capacity to survive in the environment, then more virulent strains with more readily cleaved HA protein might be less capable of surviving in the environment. Thus, selection for the capacity of virus to maintain its uncleaved form outside the host may provide a mechanism for limiting the virulence of some types of viruses.

Components of cells and host subverted for the replication of reoviruses and others. Viruses must subvert cellular components and processes for their replication. Because animal viruses infect complex host organisms, they may similarly commandeer components or processes unique to the host. Activation of reoviruses by extracellular proteases in the enteric tract of the host is an example of the latter. Other components and processes of cells and host utilized by reoviruses for replication include $(a)$ intestinal $\mathbf{M}$ cells, which normally transport antigenic substances from the intestinal lumen to lymphoid tissue in underlying Peyer's patches, for permitting reoviruses to cross the barrier of intestinal epithelium and gain access to the inside of the host (54); (b) components on the cell surface (sialic acid groups [62], perhaps attached to particular proteins including one similar to $\beta$-adrenergic receptor [63]) for permitting reoviruses to attach to cells as an initial step in infection; and $(c)$ fast axonal transport, by which neurons normally move factors between cell body and sites along the axon, for permitting reoviruses to spread via nerves to distant sites in the host (56).

Other animal viruses may share with reoviruses the mechanism of using extracellular proteases for activation of viral particles during entry into the host. Similarly, subversion of other components and processes of cells and host is common to reoviruses and other viruses and microbes: $(a)$ polioviruses and coronaviruses, as well as some bacterial species, can gain entry into the host via intestinal $\mathrm{M}$ cells; $(b)$ binding to specific receptors on cells is a mechanism adopted by all viruses and exemplified by binding to sialic acid by orthomyxoviruses and binding to CD4 protein by human immunodeficiency viruses; and (c) rabies virus, polioviruses, and some herpesviruses utilize fast axonal transport for spreading via nerves (6). These findings highlight the fact that different viruses and microbial agents have evolved similar strategies for overcoming obstacles to replication in their hosts and demonstrate the relevance of studies with reoviruses for characterizing fundamental steps in viral and microbial replication in the host and mechanisms of viral and microbial pathogenesis.

\section{Concluding remarks: step-specific determinants of the pathogenesis of reovirus infections}

Fig. 2 summarizes observations relating steps in the replication program of reoviruses to the different viral forms. It also integrates observations from numerous genetic and biochemical studies. We have frequently used a genetic approach to identify specific viral genes that determine phenotypic differences in strains of reoviruses (1). For example the $S 1$ gene, which encodes the cell-attachment protein $\sigma 1$, is the primary genetic determinant of tropism and injury within the central nervous system (3) and the pathway of spread within the host (56). Genetic studies have also identified the L2 and S1 genes in the capacity of reoviruses to infect mice via the enteric route (64). Results of these genetic studies make some general sense according to Fig. 2; they reflect which protein components comprise the type of viral particle that acts primarily at each step in the replication program of reoviruses. We can therefore discuss 
step-specific determinants of the pathogenesis of reovirus infections: individual viral proteins in distinct viral forms that act during viral replication in cells and host.

In closing, we suggest that reoviruses continue to offer lessons for characterizing mechanisms of viral pathogenesis. Studies with reoviruses also exemplify an important general approach, in which observations made at multiple levels of inquiry are integrated in the attempt to understand the pathogenesis of viral (and microbial) infections.

\section{Acknowledgments}

We thank Marcia Kazmierczak for assistance in preparing the manuscript and Virginia Hinshaw, David Knipe, Hermann Lisco, Lynda Morrison, and Leslie Schiff for reviewing portions of it before submission. Thanks also go to the many members of the laboratory past and present who contributed to this work via innumerable discussions.

The laboratory is supported by program project grant 2P50 NS16998 from the National Institute of Neurological and Communicative Disorders and Stroke, research grant 5R37 AI13178-13 from the National Institute of Allergy and Infectious Diseases, and the Shipley Institute of Medicine. M. L. Nibert was additionally supported by national research service award 5T32 HL07627 from the National Heart, Lung, and Blood Institute.

\section{References}

1. Tyler, K. L., and B. N. Fields. 1990. Reoviruses. In Fields Virology. 2nd ed. B. Fields and D. Knipe, editors. Raven Press, Inc., New York. 1307-1328.

2. Morrison, L. A., and B. N. Fields. 1991. Parallel mechanisms in the neuropathogenesis of enteric virus infections. J. Virol. 65:2767-2772.

3. Weiner, H. L., D. Drayna, D. R. Averill, Jr., and B. N. Fields. 1977. Molecular basis of reovirus virulence: role of the S1 gene. Proc. Natl. Acad. Sci. USA. 74:5744-5748.

4. Sherry, B., F. J. Schoen, E. Wenske, and B. N. Fields. 1989. Derivation and characterization of an efficiently myocarditic reovirus variant. J. Virol. 63:4840 4849 .

5. Murphy, B. R., and R. M. Chanock. 1990 . Immunization against viruses. In Fields Virology. 2nd ed. B. Fields and D. Knipe, editors. Raven Press, Inc. New York. 469-502.

6. Tyler, K. L., and B. N. Fields. 1990. Pathogenesis of viral infections. In Fields Virology. 2nd ed. B. Fields and D. Knipe, editors. Raven Press, Inc., New York. 191-239.

7. Tyler, K. L., H. W. Virgin IV, R. Bassel-Duby, and B. N. Fields. 1989. Antibody inhibits defined stages in the pathogenesis of reovirus serotype 3 infection of the central nervous system. J. Exp. Med. 170:887-900.

8. Morrison, L. A., R. L. Sidman, and B. N. Fields. 1991. Direct spread of reovirus from the intestinal lumen to the central nervous system through vagal autonomic nerve fibers. Proc. Natl. Acad. Sci. USA. 88:3852-3856.

9. Keroack, M., and B. N. Fields. 1986. Viral shedding and transmission between hosts determined by reovirus L2 gene. Science (Wash. DC). 232:16351638.

10. Schiff, L. A., and B. N. Fields. 1990. Reoviruses and their replication. In Fields Virology. 2nd ed. B. Fields and D. Knipe, editors. Raven Press, New York. 1275-1306.

11. Metcalf, P. 1982. The symmetry of the reovirus outer shell. J. Ultrastruct. Res. 78:292-301

12. Khaustov, V. I., M. B. Korolev, and V. N. Reingold. 1987. The structure of the capsid inner layer of reoviruses. Brief report. Arch. Virol. 93:163-167.

13. White, C. K., and H. J. Zweerink. 1976. Studies on the structure of reovirus cores: selective removal of polypeptide $\lambda 2$. Virology. 70:171-180.

14. Jayasuriya, A. K., M. L. Nibert, and B. N. Fields. 1988. Complete nucleotide sequence of the M2 gene segment of reovirus type 3 Dearing and analysis of its protein product $\mu 1$. Virology. 163:591-602.

15. Nibert, M. L., L. A. Schiff, and B. N. Fields. 1991. Mammalian reoviruses contain a myristoylated structural protein. J. Virol. 65:1960-1967.

16. Schiff, L. A., M. L. Nibert, M. S., Co, E. G. Brown, and B. N. Fields. 1988. Distinct binding sites for zinc and double-stranded RNA in the reovirus outer capsid protein sigma 3. Mol. Cell Biol. 8:273-283.

17. Bartlett, J. A., and W. K. Joklik. 1988. The sequence of the reovirus serotype $3 \mathrm{~L} 3$ genome segment which encodes the major core protein $\lambda 1$. Virology. 167:31-37.

18. Ralph, S. J., J. D. Harvey, and A. R. Bellamy. 1980. Subunit structure of the reovirus spike. J. Virol. 36:894-896.
19. Furlong, D. B., M. L. Nibert, and B. N. Fields. 1988. Sigma 1 protein of mammalian reoviruses extends from the surface of viral particles. J. Virol. 62:246-256

20. Hayes, E. C., P. W. K. Lee, S. E. Miller, and W. K. Joklik. 1981. The interaction of a series of hybridoma IgGs with reovirus particles: demonstration that the core protein $\lambda 2$ is exposed on the particle surface. Virology. 108:147-155.

21. Bassel-Duby, R., M. L. Nibert, C. J. Homcy, B. N. Fields, and D. G. Sawutz. 1987. Evidence that the sigma 1 protein of reovirus serotype 3 is a multimer. J. Virol. 61:1834-1841.

22. Fraser, R. D. B., D. B. Furlong, B. L. Trus, M. L. Nibert, B. N. Fields, and A. C. Steven. 1990. Molecular structure of the cell-attachment protein of reovirus: correlation of computer-processed electron micrographs with sequencebased predictions. J. Virol. 64:2990-3000.

23. Mah, D. C. W., G. Leone, J. M. Jankowski, and P. W. K. Lee. 1990. The $\mathrm{N}$-terminal quarter of reovirus cell attachment protein $\sigma 1$ possesses intrinsic virion-anchoring function. Virology. 179:95-103.

24. Banerjea, A. C., K. A. Brechling, C. A. Ray, H. Erikson, D. J. Pickup, and W. K. Joklik. 1988. High-level synthesis of biologically active reovirus protein $\sigma 1$ in a mammalian expression vector system. Virology. 167:601-612.

25. Nibert, M. L., T. S. Dermody, and B. N. Fields. 1990. Structure of the reovirus cell-attachment protein: a model for the domain organization of $\sigma 1 . J$. Virol. 64:2976-2989.

26. Rossman, M. G., E. Arnold, J. W. Erickson, E. A. Frankenberger, J. P. Griffith, H.-J. Hecht, J. E. Johnson, G. Kamer, M. Luo, A. G. Mosser, R. R. Rueckert, B. Sherry, and G. Vriend. 1985. Structure of a human common cold virus and functional relationship to other picornaviruses. Nature (Lond.). 317:145-153.

27. Coombs, K. M., B. N. Fields, and S. C. Harrison. 1990. Crystallization of the reovirus type 3 Dearing core. Crystal packing is determined by the $\lambda 2$ protein. J. Mol. Biol. 215:1-5.

28. Yeager, M. K. A. Dryden, N. H. Olson, H. B. Greenberg, and T. S. Baker. 1990. Three-dimensional structure of rhesus rotavirus by cryoelectron microscopy and image reconstruction. J. Cell Biol. 110:2133-2144.

29. Joklik, W. K. 1972. Studies on the effect of chymotrypsin on reovirions. Virology. 49:700-715.

30. Shatkin, A. J., and A. J. LaFiandra. 1972. Transcription by infectious subviral particles of reovirus. J. Virol. 10:698-706.

31. Borsa, J., T. P. Copps, M. D. Sargent, D. G. Long and J. D. Chapman. 1973. New intermediate subviral particles in the in vitro uncoating of reovirus virions by chymotrypsin. J. Virol. 11:552-564.

32. Borsa, J., D. G. Long, M. D. Sargent, T. P. Copps, and J. D. Chapman. 1974. Reovirus transcriptase activation in vitro: involvement of an endogenous uncoating activity in the second stage of the process. Intervirology. 4:171-188.

33. Sturzenbecker, L. J., M. Nibert, D. Furlong, and B. N. Fields. 1987 Intracellular digestion of reovirus particles requires a low $\mathrm{pH}$ and is an essential step in the viral infectious cycle. J. Virol. 61:2351-2361.

34. Ewing, D. D., M. D. Sargent, and J. Borsa. 1985. Switch-on of transcriptase function in reovirus: analysis of polypeptide changes using 2-D gels. Virology. 144:448-456.

35. Drayna, D., and B. N. Fields. 1982. Activation and characterization of the reovirus transcriptase: genetic analysis. $J$. Virol. 41:110-118.

36. Yamakawa, M., Y. Furuichi, and A. J. Shatkin. 1982. Reovirus transcriptase and capping enzymes are active in intact virions. Virology. 118:157-168.

37. Yeung, M. C., D. Lim, R. Duncan, M. S. Shahrabadi, L. W. Cashdollar, and P. W. K. Lee. 1989. The cell attachment proteins of type 1 and type 3 reovirus are differentially susceptible to trypsin and chymotrypsin. Virology. 170:62-70.

38. Chang, C.-T., and H. J. Zweerink. 1971. Fate of parental reovirus in the infected cell. Virology. 46:544-555.

39. Silverstein, S. C., C. Astell, D. H. Lewin, M. Schonberg, and G. Acs. 1972. The mechanism of reovirus uncoating and gene activation in vivo. Virology. 47:797-806.

40. Borsa, J., M. D. Sargent, P. A. Lievaart, and T. P. Copps. 1981. Reovirus: evidence for a second step in the intracellular uncoating and transcriptase activation process. Virology. 111:191-200.

41. Canning, W. M., and B. N. Fields. 1983. Ammonium chloride prevents lytic growth of reovirus and helps to establish persistent infection in mouse $\mathrm{L}$ cells. Science (Wash. DC). 219:987-988.

42. Maratos-Flier, E., M. J. Goodman, A. H. Murray, and C. R. Kahn. 1986. Ammonium inhibits processing and cytotoxicity of reovirus, a non-enveloped virus. J. Clin. Invest. 78:1003-1007.

43. Borsa, J., B. D. Morash, M. D. Sargent, T. P. Copps, P. A. Lievaart, and J. G. Szekely. 1979. Two modes of reovirus entry into L cells. J. Gen. Virol. 45:161-170.

44. Moody, M. D., and W. K. Joklik. 1989. The function of reovirus proteins during the reovirus multiplication cycle: analysis using monoreassortants. Virology. 173:437-446.

45. Lucia-Jandris, P. A. 1990. Interaction of mammalian reovirus particles with cell membranes. Ph.D. thesis. Harvard University 1-178.

46. Cleveland, D. R., H. Zarbl, and S. Millward. 1986. Reovirus guanylyltransferase is L2 gene product lambda 2. J. Virol. 60:307-311. 
47. Seliger, L. S., K. Zheng, and A. J. Shatkin. 1987. Complete nucleotide sequence of reovirus L2 gene and deduced amino acid sequence of viral mRNA guanylyltransferase. J. Biol. Chem. 262:16289-16293.

48. Morozov, S. Y. 1989. A possible relationship of reovirus putative RNA polymerase to polymerases of positive-strand RNA viruses. Nucleic Acids Res. 17:5394.

49. Weiner, H. L., R. F. Ramig, T. A. Mustoe, and B. N. Fields. 1978. Identifcation of the gene coding for the hemagglutinin of reovirus. Virology. 86:581584.

50. Weiner, H. L., K. A. Ault, and B. N. Fields. 1980. Interaction of reovirus with cell surface receptors. I. Murine and human lymphocytes have a receptor for the hemagglutinin of reovirus type 3. J. Immunol. 124:2143-2148.

51. Lee, P. W. K., E. C. Hayes, and W. K. Joklik. 1981. Protein $\sigma 1$ is the reovirus cell attachment protein. Virology. 108:156-163.

52. Bodkin, D. K., M. L. Nibert, and B. N. Fields. 1989. Proteolytic digestion of reovirus in the intestinal lumens of neonatal mice. J. Virol. 63:4676-4681.

53. Bass, D. M., D. Bodkin, R. Dambrauskas, J. S. Trier, B. N. Fields, and J. L. Wolf. 1990. Intraluminal proteolytic activation plays an important role in replication of type 1 reovirus in the intestines of neonatal mice. J. Virol. 64:18301833.

54. Wolf, J. L., D. H. Rubin, R. Finberg, R. S. Kauffman, A. H. Sharpe, J. S. Trier, and B. N. Fields. 1981. Intestinal M cells: a pathway for entry of reovirus into the host. Science (Wash. DC). 212:471-472.

55. Rubin, D. H., M. J. Kornstein, and A. O. Anderson. 1985. Reovirus serotype 1 intestinal infection: a novel replicative cycle with ileal disease. J. Virol. 53:391-398.
56. Tyler, K. L., D. A. McPhee, and B. N. Fields. 1986. Distinct pathways of viral spread in the host determined by reovirus S1 gene segment. Science (Wash. DC). 233:770-774.

57. Drayna, D., and B. N. Fields. 1982. Genetic studies on the mechanism of chemical and physical inactivation of reovirus. J. Gen. Virol. 63:149-160.

58. Finlay, B. B., and S. Falkow. 1989. Common themes in microbial pathogenicity. Microbiol. Rev. 53:210-230.

59. Payne, C. C., and P. P. C. Mertens. 1983. Cytoplasmic polyhedrosis viruses. In The Reoviridae. W. Joklik, editor. Plenum Publishing Corp., New York 425-504.

60. Chow, M., J. F. E. Newman, D. Filman, J. M. Hogle, D. J. Rowlands, and F. Brown. 1987. Myristylation of picornavirus capsid protein VP4 and its structural significance. Nature (Lond.). 327:482-486.

61. Murphy, B. R., and R. G. Webster. 1990. Orthomyxoviruses. In Fields Virology. 2nd ed. B. Fields and D. Knipe, editors. Raven Press, Inc., New York. 1091-1153.

62. Paul, R. W., A. H. C. Choi, and P. W. K. Lee. 1989. The $\alpha$-anomeric form of sialic acid is the minimal receptor determinant recognized by reovirus. Virology. 172:382-385.

63. Co, M. S., G. N. Gaulton, A. Tominaga, C. J. Homcy, B. N. Fields, and M. I. Greene. 1985. Structural similarities between the mammalian beta-adrenergic and reovirus type 3 receptors. Proc. Natl. Acad. Sci. USA. 82:5315-5318.

64. Bodkin, D. K., and B. N. Fields. 1989. Growth and survival of reovirus in intestinal tissue: role of the L2 and S1 genes. J. Virol. 63:1188-1193. 